\title{
UN POSIBLE ENFOQUE DE COMPLEMENTARIEDAD ENTRE ESPACIOS DE TRABAJO MATEMÁTICO Y MODELIZACIÓN
}

\section{A POSSIBLE COMPLEMENTARITY APPROACH BETWEEN MATHEMATICAL WORKING SPACES AND MODELING}

\section{PAULA VERDUGO-HERNÁNDEZ*}

Rec.: 05-11-2019. Acept.: 23-10-2020. Publ.: 18-12-2020

DOl: http://doi.org/10.29035/ucmaule.59.12

\section{RESUMEN}

Este artículo presenta un estudio sobre una posible complementariedad entre los Espacios de Trabajo Matemático (ETM) y el enfoque de modelización de Blomhøj. Desde una perspectiva teórica que provee fundamentos con el fin de conectar teorías en educación matemática, se pretende extender la discusión sobre los alcances de la complementariedad y se ilustra mediante un conocido ejemplo propuesto por Blomhøj. El resultado principal muestra que ambos marcos teóricos se complementarían incluso en la dimensión epistemológica, característica que distinguiría el ciclo de Blomhøj de otros que enfatizan principalmente la dimensión cognitiva de la modelización, aspecto que enriquecería el análisis de este tipo de tareas, ampliando la articulación entre ambos marcos teóricos, cuestión que hasta donde se sabe, no ha sido realizada hasta ahora.

Palabras clave: complementariedad, modelización, espacios de trabajo matemático, ciclo de Blomhøj.

* Universidad Adventista de Chile, Chillán, Chile. https://orcid.org/0000-0001-6162-654X paulasinttia@gmail.com 


\section{ABSTRACT}

This article presents a study on a possible complementarity between Mathematical Working Spaces (MWS) and Blomhøj's modeling approach. From a theoretical perspective that provides foundations to connect theories in mathematics education, it intends to extend the discussion on the scope of complementarity and illustrates it by a well-known example proposed by Blomhøj. The main result shows that both theoretical frameworks would complement each other even in the epistemological dimension, characteristic that would distinguish the Blomhøj cycle from others that mainly emphasize the cognitive dimension of modeling. This aspect would enrich the analysis of this type of tasks, expanding the articulation between both theoretical frameworks, a question that, as far as it knows, has not been done so far.

Key words: Complementarity, Modeling, Mathematical Working Spaces, Blomhøj' cycle.

\section{INTRODUCCIÓN}

La teoría de los Espacios de Trabajo Matemático (ETM) es un marco analítico general, concebido para estudiar el trabajo matemático a partir de la resolución de problemas en un contexto educativo, que ha sido construido y aplicado en diversas investigaciones dentro de la didáctica de la matemática, en diversos países tales como Francia, Canadá y España (Kuzniak, 2011; Gómez-Chacón et al., 2016; Kuzniak, Nechache \& Drouhard, 2016; Verdugo-Hernández, 2020). Por otra parte, la modelización matemática es una teoría que emergió del estudio de los procesos de enseñanza y aprendizaje de la matemática y la cual hasta el presente no tiene una única perspectiva, pero que sin embargo, puede ser delimitada mediante puntos de vista consensuados (Blomhøj, 2004). Dicha teoría aborda específicamente el proceso de modelización que debería ser conducido para la resolución de determinados problemas que requieren este enfoque. A saber, problemas de modelización, los cuales constituyen una categoría dentro de los problemas matemáticos, y el cual ha sido ampliamente estudiado por comunidades e investigaciones científicas que lo han posicionado en los currículos del mundo (Kaiser, 1995), destacando los trabajos de Blum y colaboradores (e.g., Blum \& Leiß, 2007), considerados referentes reconocidos dentro de los enfoques de modelización tradicionales. Es posible encontrar varios 
trabajos que analizan la modelización mediante el ETM, encontrando diversos puntos de vista: algunos abordan tareas de modelización como un entorno contextualizado para activar el ETM (Rauscher \& Adjiage, 2014); otros analizan los efectos de reconocer la realidad como un entorno singular para la activación del ETM (Parzycs, 2014). Los dos primeros grupos de trabajos aplican exclusivamente el ciclo de Blum y Leiß (Blum \& Leiß, 2007) para analizar el trabajo matemático derivado de la resolución de tareas de modelización en términos del ETM, sin cuestionarse los aspectos teóricos del proceso, y sin intentar complementar ambos constructos teóricos. De particular interés en el presente artículo es el grupo de trabajos que reflexionan sobre diversas concepciones de modelización con el fin de adaptar el análisis al campo de las probabilidades mediante el ETM (Nechache, 2016; Derouet 2016). En este escrito, se invita a extender los puntos de vista anteriormente descritos, enfocándose en otra corriente de modelización matemática, y no necesariamente pensando en un campo específico de la matemática, con el objetivo de establecer otras formas de análisis descriptivo de tareas de modelización, aportando a una posible articulación entre el ETM y la modelización. Para ello, se toman en cuenta ciertos aspectos teóricos que permitirían esta complementación (e.g., Radford, 2008), iniciando así un estudio sistemático de este tema (articulación ETM-modelización), cuestión que, hasta donde se sabe, no ha sido conducida. Específicamente, en este artículo se pretende complementar el ETM con el ciclo de Blomhøj, debido a que éste permite ampliar el análisis de tareas de modelización mediante el ETM, dado que el ciclo considera una dimensión epistemológica de la modelización, aspecto que no es tomado en cuenta por el ciclo de Blum y Leiß, y el cual es importante desde el punto de vista del ETM. Cabe señalar que no se espera reconocer la modelización adecuada para el ETM, sino incentivar estudios sobre complementación entre ambas teorías, despojándose de la unicidad conceptual, en el sentido de considerar solamente el ciclo de Blum y Leiß en el ETM, y reconocer fortalezas en diferentes enfoques teóricos de modelización, en el sentido de su complementación con variados aspectos del ETM, y no solamente con la dimensión cognitiva.

\section{Revisión de Literatura}

A continuación, haremos una breve revisión de trabajos de modelización en ETM, cuya discusión está actualmente latente y en desarrollo. De hecho, en el Sexto 
Simposio sobre el Trabajo Matemático (ETM6), llevado a cabo en diciembre de 2018 en Valparaíso, Chile, se han reportado enfoques teóricos con una clara tendencia hacia la aproximación individualizada de la modelización, destacando estudios descriptivos e interpretativos respecto a la puesta en práctica de tareas de modelización en el aula, la activación de planos y circulaciones existentes en el ETM, y la posibilidad de diseñar tareas de modelización basadas en problemas genuinamente reales. En general, las investigaciones de modelización en ETM se basan en metodologías cualitativas y empíricas, centradas en la interacción del mundo real $u$ otras ciencias con los modelos matemáticos. A continuación, discutimos brevemente algunos de estos trabajos, que son de interés para la presente investigación.

Derouet (2016) analiza varios ciclos de modelización, Kaiser (1995), Blum (1996), Blum \& Leiß (2007), resaltando aquellos de Coulange (1998) y Henry (2001), ya que utilizan el dominio pseudo-concreto como parte de un dominio matemático, considerando las elecciones hechas sobre el modelo en la realidad. La autora se inclina por el ciclo de modelización de Blum y Leiß dado que, según su argumentación, las etapas propuestas por Henry (2001), quien propone un ciclo adaptado al campo de las probabilidades, son representadas como acciones o estados cuyos estatus dependen del objetivo de la tarea que se desea resolver, dando lugar a cierta ambigüedad.

Por otra parte, Nechache (2018) estudia distintos enfoques de modelización, dado que es necesario construir modelos para la resolución de tareas en el campo de las probabilidades, lo cual demanda incorporar diferentes etapas del proceso de modelización, induciendo usos o cambios de paradigmas probabilistas (Parzysz, 2011) y de dominios (Montoya \& Vivier, 2016) en el marco del ETM. Adicionalmente, la autora propone un enfoque de modelización, adaptado al campo de las probabilidades (Nechache, 2016), sobre la base de los ciclos establecidos por Kaiser (1995), Blum \& Leiß (2007), Borromeo-Ferri (2006) y Henry (1997), adaptando tres etapas fundamentales del ciclo del último autor: descripción de la realidad, matematización y validación externa. De acuerdo a la autora, en la primera etapa es necesario plantear hipótesis, siendo pertinente desarrollar el ETM de referencia del campo en estudio, previamente a la construcción del modelo matemático. De lo anterior, se desprende que el ETM puede estar presente en las diversas etapas de la modelización, y no solamente después de la construcción del modelo. 
De la revisión anterior, se desprende que existe la necesidad de realizar trabajos que aporten sistemáticamente a la discusión sobre la complementariedad entre ETM y modelización. Para ello, habría que tomar en cuenta aspectos teóricos requeridos para tal efecto (e.g, ver Radford, 2008); por otro lado, prácticamente todos se limitan al análisis de tareas en base al ETM, usando el ciclo de Blum y Leiß para estudiar las diversas etapas del proceso de modelización, y excepcionalmente a partir de otros ciclos (Nechache, 2016, 2018, adapta el ciclo de Henry, el cual pareciera más adaptado para la enseñanza de las probabilidades a nivel secundario).

De acuerdo con la argumentación desarrollada hasta ahora, en este artículo se desea abordar sistemáticamente la complementariedad entre ETM y modelización, tomando en cuenta los aspectos teóricos sugeridos por Radford (2008). Para lo anterior, se considera el enfoque de Blomhøj ya que, como se verá a continuación, éste posee aspectos interesantes que lo distinguen del enfoque de Blum y Leiß, y que son compatibles con el ETM, en términos de aquello que es considerado esencial por Radford (2008) para conectar teorías en educación matemática; particularmente, el hecho que el enfoque de Blomhøj considera una dimensión epistemológica en el proceso de modelización, aspecto esencial para el ETM, como se verá en el desarrollo de esta sección. De este modo, el presente trabajo podría ser el punto de partida para estudiar la complementariedad entre ambos marcos teóricos, lo que permitiría ampliar el análisis de tareas de modelización mediante el ETM, eventualmente propiciando mejores diseños que promuevan un proceso adecuado de enseñanza y aprendizaje en torno a este tipo de tareas, sobre lo cual no existe consenso.

\section{Marco Teórico}

En las subsecciones Ciclo de modelización matemática de Blomhøj y Espacios de Trabajo Matemático se describen el ciclo de modelización de Blomhøj y el ETM, respectivamente. En la subsección Complementariedad entre el ETM y el ciclo de modelización de Blomhøj, se pretende construir el anunciado enfoque de complementariedad entre ambas teorías, teniendo en cuenta dos factores importantes. Primero, aquellos aspectos teóricos que, a juicio de Radford (2008), permitirían establecer adecuadamente una conexión entre teorías de educación matemática. Segundo, se contrasta el ciclo de Blomhøj con el de Blum y Leiß con el fin de resaltar las fortalezas del primero, relativo a su complementación con el 
Verdugo, P. (2020). Un posible enfoque de com plem entariedad entre espacios de trabajo matem ático y modelización. UCMaule, 59, julio-diciem bre, 12-30. DOI: http://doi.org/10.29035/ucm aule.59.12

ETM, y para destrabar la unicidad teórica evidenciada hasta ahora, en el sentido de usar casi exclusivamente el enfoque de Blum y Leiß en el contexto del ETM.

\section{Ciclo de modelización matemática de Blomhøj}

Desde un enfoque tradicional del pensamiento, se plantea la modelización desde un carácter principalmente multidisciplinario, prevaleciendo un proceso de investigación dinámico, considerando como base las relaciones entre los conceptos matemáticos, ideas y experiencias de la vida real (Artigue \& Blomhøj, 2013). Blomhøj (2004) reconoce la modelización matemáticacomo un proceso esquematizadoa partir de un enfoque cíclico para una aproximación individualizada. De esta manera, el ciclo de modelización de Blomhøj parece similar al de Blum y Leiß (Doerr, Ärlebäck \& Misfeldt, 2017), sin embargo, el primero supone una visión distintiva para la articulación entre el saber matemático y la conexión con la realidad o dominio de investigación del problema que se desea resolver, ya que el modelo matemático es visto como un conector entre el sistema matemático y la situación contextualizada de investigación, con distinción de propósitos. En efecto, los objetivos del dominio de investigación implicarían un modelo descriptivo, explicativo o predictivo (Doerr et al., 2017), o viceversa, dependiendo del alcance de un modelo se pueden clarificar los objetivos. Tales alcances, según Blomhøj (2004), caracterizan las dificultades epistemológicas que surgen, ya que en un alcance predictivo se requieren mayores supuestos e hipótesis, de lo cual la práctica educativa ha evidenciado una mayor complejidad (Blomhøj, 2004; Blum \& Borromeo-Ferri, 2009). Esta característica condujo a visualizar el ciclo de Blomhøj como una descripción de competencias en matemática, reconociendo la modelización matemática como una competencia diferenciada (Blomhøj \& Jensen, 2007).

Para Blomhøj (2004), el proceso de modelización inicia desde un objeto del mundo real, en un contexto conocido por el estudiante, lo que lleva a la formulación que realmente solicita el problema. Por ejemplo, considerando el proyecto de enseñanza "Mañanas Matemáticas" (ver referencia citada), cuyo problema es el ahorro de agua en las mañanas, quizás la pregunta sería cómo optimizar ciertas variables en la ducha tales como el flujo de agua y duración de la misma, lo cual no necesariamente tiene por qué ser enunciado explícitamente. Esto guiará el abordaje de la pregunta hacia el dominio de investigación en donde se posiciona el desarrollo propuesto (el que no es 
único). A continuación, existe una necesidad por la selección de variables relevantes (e.g., flujo de agua y duración de la ducha, vistos como variables matemáticas), relaciones (e.g., entre la cantidad de agua consumida y el tiempo usado en la ducha), objetos (e.g., la variables físicas flujo de agua y duración de la ducha, antes de traducirlas en variables mediante lenguaje matemático), apuntando hacia una idealización del problema, la cual entregará indicios adecuados para la traducción de variables, objetos o relaciones en un lenguaje formal, delimitando el trabajo matemático. Esto permitirá que el proceso avance hacia un sistema matemático, conduciendo al modelo, en donde tanto los métodos matemáticos como su lógica hipotética deductiva actúan en el análisis, logrando establecer los resultados del modelo. Tales deducciones son interpretadas a partir del dominio de investigación utilizado, logrando proyectar la capacidad de ganar un adecuado entendimiento y profundidad, para finalmente validarlos a partir de los datos observados, el conocimiento teórico o la experiencia personal o compartida. El dinamismo del ciclo de modelización es diagramado en la Figura 1.

(f) Validación

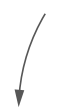

Acción/Insight

(e) Interpretación/Evaluación

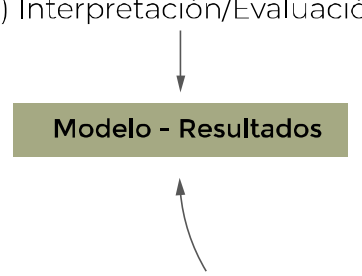

(d) Análisis del sistema matemático

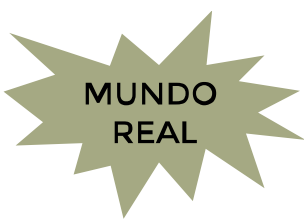

(a) Formulación del problema

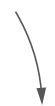

\section{Dominio de investigación}

(b) Sistematización

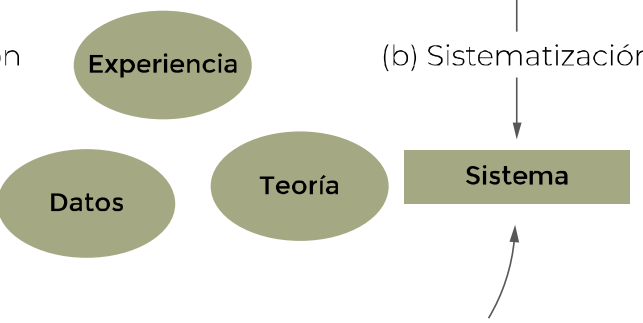

\section{Sistema matemático}

Figura 7. Traducido del ciclo de modelización de Blomhøj (Blomhøj \& Kjeldsen, 2006). 
En el ciclo de Blomhøj (Figura 1), la experiencia, los datos y la teoría son planteados como la base para todos sus subprocesos, (a)-(e). La teoría proviene del conocimiento sobre el dominio de investigación usado en el proceso de modelización, la cual podría tener un estatus epistemológico bastante diferente, aún dentro del ciclo, incluyendo teorías debidamente fundamentadas como la física. Cuando este estatus no es tan claro (o, al menos, no lo es para el resolutor del problema), el conocimiento provendría de la experiencia personal o compartida, e incluso de suposiciones ad hoc. Este conocimiento teórico es fundamental para determinar cómo el modelo y sus eventuales aplicaciones pueden ser validados. En este sentido, los datos, previos al proceso, podrían ser usados en la sistematización y matematización, y eventualmente para validar el modelo. El uso de los datos en la sistematización y matematización implica una construcción inductiva del modelo, aunque los modelos matemáticos que ya existen en la teoría, invitan a su uso en la resolución de tareas de modelización, propiciando una actividad deductiva.

Después de recorrer un proceso de modelización, cualquiera que sea, el producto obtenido es un modelo matemático, el cual abarca, según Blomhøj (2004), un conjunto de relaciones entre ciertos objetos matemáticos y sus conexiones, y una situación o fenómeno de naturaleza no matemática, definición que tiene implicancias didácticas fundamentales. Por un lado, todo modelo matemático está asociado, explícita o implícitamente, con una situación extramatemática; $y$, por otro lado, para que un sujeto resolutor (e.g., el estudiante) pueda trabajar apropiadamente con un modelo matemático, el prerrequisito es que éste descubra la situación o fenómeno que se desea modelizar y la matemática que está detrás, como dos objetos separados, pero a la vez interconectados.

En síntesis, los ciclos de Blum \& Leiß (2007) y Blomhøj (2004) coinciden en que ambos consideran al proceso de modelización matemática como un puente entre la realidad del que resuelve la tarea y la matemática, visualizando el proceso de modelización como una tendencia de relevancia para la educación matemática (Kaiser, Blum, Borromeo-Ferri, \& Stillman, 2011). Sin embargo, en el ciclo de Blomhøj es posible precisar tanto el dominio de investigación como el sistema que define el estudiante, siendo una característica que permite predecir — desde el punto de vista de la investigación — variadas formas de sistematización, proveyendo diversas posibilidades de construcción de modelos matemáticos. 
Esta etapa, junto con el propósito de la tarea de modelización, permitirá establecer formas de circular por el ciclo cuando se active el trabajo matemático del estudiante. Más aun, de la discusión anterior, el ciclo de Blomhøj permitiría incorporar aspectos epistemológicos del conocimiento teórico del dominio de investigación, que se retroalimenta con la formulación del problema, sistematización y matematización. De este modo, hay un impacto en el proceso completo de modelización, posibilitando abarcar no solamente la perspectiva cognitiva, subyacente al ciclo de Blum y Leiß, sino que además una perspectiva epistemológica (que éste último ciclo no enfatiza), cuestión muy importante para la complementariedad del ciclo de Blomhøj con el ETM, el cual abarca ambas dimensiones, epistemológica y cognitiva, tal como se discutirá a continuación.

\section{Espacios de Trabajo Matemático}

Los Espacios de Trabajo Matemático (ETM) (Kuzniak, 2011) es un marco teórico que tiene por objetivo principal estudiar el trabajo matemático en un contexto educativo, con el fin de favorecer y mejorar las condiciones en las cuales se produce el proceso de enseñanza-aprendizaje de la matemática. Para definir el ETM se introducen dos planos horizontales, uno epistemológico y otro cognitivo, los cuales poseen tres componentes cada uno. El plano epistemológico está constituido del referencial (formado por las propiedades, teoremas y definiciones), el representamen (conjunto de signos), y los artefactos (materiales o simbólicos). El plano cognitivo está constituido por los polos de visualización (representación del espacio y al soporte material), construcción (instrumentos y técnicas asociadas) y prueba (proceso relativo a la validación). Los planos están conectados mediante distintas génesis: la génesis instrumental, que permite hacer operatorios a los artefactos en el proceso constructivo; la génesis semiótica, basada en los registros de representación semióticos, que asegura a los objetos tangibles su estatus de objetos matemáticos operatorios; y la génesis discursiva de la prueba que da sentido a las propiedades para ponerlas al servicio del razonamiento matemático. 


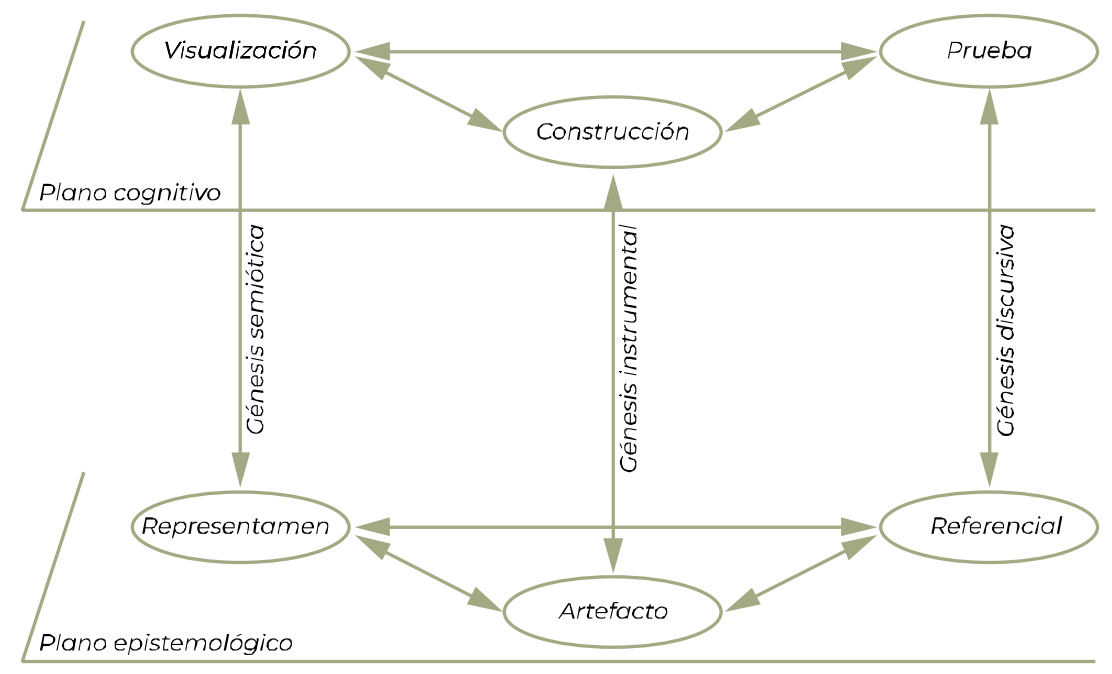

Figura 2. ETM y sus génesis (Kuzniak, 2011)

Según Kuzniak \& Richard (2014), el marco ETM considera tres planos verticales, los cuales se activan por medio de la resolución de una determinada tarea, cada uno definido por la articulación de dos génesis: Semiótico- Instrumental [Sem-Ins]; Semiótico-Discursiva [Sem-Dis] e Instrumental-Discursivo [Ins-Dis] (ver Figura2).

Todo lo anterior permite al ETM apoyar la comprensión del modelo del trabajo matemático que se genera. Además, se identifican tres tipos de ETM: el ETM de referencia, del cual depende la organización esperada del trabajo y que se define solo sobre la base de criterios matemáticos; el ETM idóneo, que consiste en el acondicionamiento y organización del ETM de referencia, con el fin de presentarlo en una institución educativa dada con una función definida; y el ETM personal, que reside en la manera en que el ETM idóneo es utilizado por los estudiantes y también por sus profesores. Cada individuo se apropia y ocupa su propio ETM personal con sus conocimientos matemáticos y sus capacidadescognitivas (Kuzniak, 2011).

El ETM depende del dominio matemático bajo estudio, el cual guía el trabajo matemático de acuerdo con los paradigmas propios de éste. Por la naturaleza del objeto matemático considerado y por el nivel en el cual los docentes enseñan, es esperable que se pueda observar la presencia de varios paradigmas en la resolución de una tarea. 


\section{Complementariedad entre el ETM y el ciclo de modelización de Blomhøj}

Con el fin de fundamentar una posible complementariedad entre el ETM y el ciclo de modelización de Blomhøj, nos hemos basado en el trabajo de Radford (2008), el cual trata sobre los desafíos y posibilidades que ofrece la conexión de teorías en educación matemática. Al respecto, Radford señala que una conexión depende de, al menos, la estructura de las teorías y del propósito de tal conexión. El autor parte de la base que una teoría en educación matemática debe tener un objetivo descriptivo con el fin de mejorar la comprensión de los fenómenos estudiados, y un objetivo normativo para desarrollar un diseño instruccional. A partir de lo anterior, Radford conceptualiza una teoría como una forma de producir comprensiones y modos de acción basadas en un sistema de principios básicos, en una metodología y en un conjunto de preguntas de investigación paradigmáticas. Esquemáticamente, una teoría está compuesta por principios, una metodología y preguntas de investigación paradigmáticas. El sistema de principios básicos corresponde a un conjunto de proposiciones que delinean la frontera de lo que sería objeto de discusión y del enfoque de investigación de la teoría. La metodología consiste en las técnicas de recolección de datos (en un sentido amplio) y su análisis basado en el sistema de principios. El conjunto de preguntas de investigación paradigmáticas se refiere a esquemas para generar preguntas específicas en la medida que surgen nuevas interpretaciones o los principios se profundizan, amplían o modifican.

Para Radford, el objetivo de conceptualizar las teorías como el triplete principios, metodología y preguntas de investigación paradigmáticas se debería a que la conexión de teorías se produciría en varios niveles del triplete, pudiendo relacionar principios, métodos o preguntas de investigación, o una combinación de éstos. Asimismo, serviría para delimitar la conexión de teorías, llegando al punto de que algunas teorías parecieran incompatibles a primera vista.

Por otro lado, Radford asegura que, dependiendo del objetivo, las conexiones de teorías pueden tomar variadas formas, por ejemplo, comparar y contrastar, coordinar y combinar, integrar localmente y sintetizar. Con el fin de aportar una mayor comprensión de las etapas de modelización, enriqueciendo el análisis del trabajo matemático que se deriva durante el desarrollo de estas, de manera de promover a futuro un mejor diseño instruccional. El principal objetivo de la presente investigación es establecer otras formas de análisis descriptivo de tareas 
de modelización mediante el ETM, a la luz del ciclo de Blomhøj. En este sentido, no sólo se estaría destrabando la unicidad evidenciada hasta ahora, toda vez que en casi todas las investigaciones conducidas mediante el ETM se emplea el ciclo de Blum y Leiß para analizar tareas de modelización (salvo quizás en probabilidades, como ya discutimos anteriormente), sino que también se estaría enriqueciendo el ETM, incorporando aspectos importantes desde el punto de vista de éste último, y que podrían aportar al estudio del trabajo matemático en este contexto.

En cuanto a la complementariedad del ETM con el ciclo de Blomhøj, y en concordancia con la argumentación de Radford, se considera el ETM como metodología general de análisis de resolución de problemas, identificando para ese propósito las componentes epistemológicas y cognitivas movilizadas, junto a las circulaciones de conocimientos (planos verticales) que se activan en el trabajo matemático (ver subsección Espacios de Trabajo Matemático). Por su parte, el ciclo de Blomhøj es considerado como el sistema de principios que fundamentan las etapas de modelización matemática, ésta última vista como un tipo de resolución de una clase particular de problemas (los de modelización) (ver subsección Ciclo de modelización matemática de Blomhøj). Adicionalmente, se ha contrastado el ciclo de Blomhøj con el de Blum y Leiß, enfatizando aquello que consideramos deseable del primero, lo cual será retomado en el estudio teórico específico que se realizará sobre la complementariedad entre este y el ETM. Dicha conexión parece posible, debido a la generalidad de los principios del ciclo de Blomhøj, y dado que la pregunta de investigación del ciclo se relacionaría con los procesos de modelización, o más generalmente, de resolución de tareas estudiados bajo el ETM, a la luz del ciclo. Por supuesto, el ciclo de Blum y Leiß también se podría complementar con el ETM, sin embargo, se piensa abrir, al menos, el debate para conectar otros ciclos con el ETM, como el considerado aquí, y dada la riqueza que posee, como se discutió anteriormente.

Complementando lo anterior, el hecho que el fenómeno modelizado y la matemática en juego están indisolublemente unidos en el ciclo de Blomhøj, tiene consecuencias para el análisis mediante el ETM, dado que esto permitiría establecer el trabajo matemático incluso antes de la etapa de matematización, donde éste ya está claramente definido, pudiendo ser identificado y concebido desde las etapas anteriores, formulación del problema y sistematización. De esta manera, dado que el dominio de investigación y la matemática están unidos a 
Verdugo, P. (2020). Un posible enfoque de com plem entariedad entre espacios de trabajo matem ático y modelización. UCMaule, 59, julio-diciem bre, 12-30. DOI: http://doi.org/10.29035/ucm aule.59.12

través del fenómeno modelizado, el ETM puede ser establecido desde el inicio del proceso de modelización, aunque en un nivel de trabajo diferente que aquel que podría evidenciarse a partir de la etapa de matematización. En ese sentido, la teoría, como base para el ciclo de Blomhøj (ver Figura 1), tendría relación con el ETM, del hecho que la primera proviene del conocimiento del dominio de investigación, el cual está unido a la matemática, como se mencionó antes. Asimismo, los datos, también a la base del ciclo (ver Figura 1), que podrían extraerse del mundo real, pueden ser considerados como signos, los cuales pueden ser percibidos mediante la activación de la génesis semiótica en el ETM.

Dado que este artículo trata de complementar dos teorías en educación matemática, a continuación se ilustrará mediante un ejemplo cómo se podría conducir dicha conexión. Concretamente, se estudiará lo anterior a partir del ejemplo las "Mañanas Matemáticas" (Blomhøj, 2004). Previo a la comprensión del fenómeno que se desea modelizar, en el proceso de formulación del problema (Fig. 1), el resolutor estaría implícitamente activando el ETM toda vez que tendría que pensar en cómo optimizar el flujo de agua y la duración de la ducha, si la pregunta fuera ¿cómo ahorrar agua en las mañanas?, guiando el posicionamiento del problema en un referencial teórico. En la etapa de sistematización del ciclo, habría que seleccionar las variables, relaciones y objetos relevantes que, en este caso, corresponderían a objetos físicos y sus relaciones, lo cual proporcionaría los elementos adecuados para su representación en un lenguaje matemático, delimitando el ETM. Dichas variables, que representan objetos físicos (flujo y tiempo), serían internalizadas activando la génesis semiótica, construyendo una instanciación de ellas mediando la génesis instrumental, con posible exploración experimental para establecer los supuestos, generando una circulación de conocimientos en el plano vertical [Sem-Ins] del ETM. En concordancia con lo anterior, en la etapa de matematización del ciclo, se llevaría a cabo la construcción de un modelo matemático mediante el cual se relacionan las variables identificadas, concretando el proceso de sistematización, interviniendo directamente el ETM. Para ello, se movilizan las relaciones, variables y supuestos del proceso anterior, activándose varias componentes, génesis y planos verticales del ETM: el plano vertical [Sem-Ins] para el proceso de construcción y formalismo matemático (por ejemplo, la introducción simbólica de las variables), junto a componentes del plano epistemológico tales como propiedades teóricas del 
dominio matemático (por ejemplo, la cantidad de agua usada en la ducha es igual al flujo de agua por el tiempo) u otras pertinentes a la construcción del modelo.

En el proceso de análisis del sistema matemático, se activa explícitamente el ETM mediante la génesis instrumental para construir resultados matemáticos del modelo, usando herramientas matemáticas tales como métodos algorítmicos de resolución (por ejemplo, de una ecuación algebraica o diferencial); simulación en software, en caso de que el sistema matemático no tenga solución analítica o ellas sean demasiado complicadas de evaluar; tabulación de valores (por ejemplo, dar diferentes valores del tiempo o del flujo de agua para calcular la cantidad de agua usada en la ducha), por mencionar algunas. Cabe señalar que, cuando el modelo es complejo, habría activación de la génesis discursiva del ETM con el fin de demostrar la existencia, unicidad, entre otras propiedades, de las soluciones del modelo, generando conocimientos en el plano vertical [Ins-Dis] del ETM. En el caso del ejemplo que se analiza aquí, hemos considerado el modelo no complejo y una construcción de valores tabulados podría bastar para su resolución, no siendo necesario movilizar la génesis discursiva. El proceso de interpretación y evaluación considera un cuestionamiento del trabajo realizado, pudiendo retomar la teoría del dominio de investigación, volviendo a complementarse con la componente del referencial del ETM, incluso dependiendo de los resultados se podría volver a cuestionar la sistematización realizada. La interpretación de los resultados se hace en relación con el dominio de investigación, observando si hay compatibilidad con éste (por ejemplo, valores negativos de la cantidad de agua no tiene sentido), lo cual podría estar relacionado con la prueba o razonamiento matemático, activando la génesis discursiva, o con procesos algorítmicos activando la génesis instrumental. Asimismo, varias componentes del plano epistemológico y cognitivo podrían activarse (referencial, representamen y artefactos como software; y visualización y prueba, respectivamente). Por último, el proceso de validación se realizaría por comparación con datos observados u obtenidos por medio de la experiencia personal o compartida, lo cual activaría procesos en el ETM similares a aquellos de la etapa anterior. Por supuesto, la complementación del ciclo de Blomhøj y el ETM depende de la complejidad del problema 
planteado, del conocimiento o experiencia previa del resolutor, y de si el modelo es descriptivo o predictivo.

\section{Conclusiones}

El presente trabajo pretende contribuir a la discusión teórica sobre la complementariedad entre el ETM y la modelización, a partir del ciclo de Blomhøj, el cual tendría ventajas y similitudes comparado a otros ciclos, como el de Blum y Leiß.

Teniendo en cuenta que el ciclo de Blum y Leiß se ha construido principalmente para la educación escolar secundaria partiendo de un enfoque cognitivo, el ciclo de Blomhøj podría considerarse mayormente para el trabajo con modelos en el nivel de enseñanza universitaria, pues como se mencionó, este considera una componente epistemológica, que permitiría complementarlo con el ETM, enriqueciendo el análisis de tareas de modelización. En efecto, para Blomhøj, el dominio de investigación en el cual se formula el problema tiene vital importancia para el ciclo, lo cual se relacionaría directamente con la dimensión epistemológica del ETM, más aún si se involucra en el estudio de la resolución problemas genuinamente reales, aspecto considerado deseable en este marco teórico.

Si bien este trabajo se ha realizado de forma teórica, una aplicación interesante sería conducir un análisis experimental sobre tareas de modelización, enfatizando el proceso de resolución de los estudiantes (su ETM personal) a la luz del ciclo de Blomhøj, lo cual se abordará en una investigación futura.

Finalmente, otro aspecto importante del ETM corresponde a los paradigmas del campo matemático al cual pertenece el problema que se desea resolver. Definidos a partir de aspectos epistemológicos y educativos, los paradigmas guían el trabajo matemático y su estudio podría enriquecer el análisis de tareas de modelización en base al ETM, profundizando la conexión de éste con el ciclo de Blomhøj.

\section{Financiamiento}

Proyectos de Investigación Regular (2019-113). Universidad Adventista de Chile. 


\section{REFERENCIAS BIBLIOGRÁFICAS}

Artigue, M., \& Blomhøj, M.(2013). Conceptualising inquiry-based education in mathematics. ZDM - The International Journal on Mathematics Education, 45(6), 797-810.

Borromeo-Ferri, R. (2006). Theoretical and empirical differentiations of phases in the modelling process. ZDM - The International Journal on Mathematics Education, 38(2), 86-95. DOI: https://doi.org/10.1007/BF02655883

Blomhøj, M. (2004). Mathematical modelling a theory for practice. In B. Clarke, D. M. Clarke,

G. Emanuelsson, B. Johansson, D. V. Lester, A. Wallby, \& K. Wallby (Eds.), International Perspec- tives on learning and teaching mathematics (pp. 145-159). Göteborg University: National Center for Mathematics Education.

Blomhøj, M., \& Kjeldsen, T.H. (2006). Teaching mathematical modeling through project work. ZDM, 38, 163- 177. DOI: http://doi:10.1007/BF02655887

Blomhøj, M., \& Jensen, T.H. (2007). What's all the Fuss about Competencies? En: Blum W., Galbraith P.L., Henn HW., Niss M. (eds) Modelling and Applications in Mathematics Education. New ICMI Study Series, vol 10. Springer, Boston, MA. DOI: https://doi.org/10.1007/978-0-387-29822-1_3

Blum, W. (1996). Anwendungsbezuge im Mathematikunterrichtin der didaktischen Diskussion. Mathematische Semesterberichte, 32, 195-23.

Blum, W., \& Leiß, D. (2007). How Do Students and Teachers Deal with Mathematical Modelling Problems? The Example Sugaloaf und the DISUM Project. En C. Haines, P. L. Galbraith, W. Blum, \& S. Khan (Eds.), Mathematical Modelling (ICTMA72)-Education, Engineering and Economics (pp.222-231). Horwood. 
Blum, W., \& Borromeo-Ferri, R. (2009). Mathematical Modelling: Can It Be Taught And Learnt? Journal of Mathematical Modelling and Application, 7(1), 45-58.

Coulange, L. (1998). Les problèmes "concrets" à "mettre en equation" dans l'enseignement. Petitx. 47, 3-58.

Gómez-Chacón, I., Kuzniak, A., \& Vivier, L. (2016). El rol del profesor desde la perspectiva de los espacios de trabajo matemático. Boletín de Educación Matemática, Bolema, 30(54), 1-22. DOI: https://doi.org/10.1590/1980$4415 v 30 n 54 \mathrm{aO} 1$

Derouet, $\mathrm{CH}$.(2016). La fonction de densité au carrefour entre probabilités et analyse en terminale S. Tesis Doctoral. Universidad de Paris Diderot (Paris 7), Francia. Recuperado de http://theses.md.univ- parisdiderot.fr/DEROUET_charlotte_2_va_20161125.pdf

Doerr, H., Ärlebäck, J., y Misfeldt, M. (2017). Representations of modelling in mathematics education. In G. Stillman, W. Blum \& G. Kaiser (Eds.), Mathematical modelling and applications: Crossing and researching boundaries in mathematics education (pp. 7182). Springer.

Henry, M. (1997). Notion de modele et modélization en l'enseignement. En: Enseigner les probabilités au lycée. Reims: Commission Inter-IREM. pp. 77-84.

Henry,M. (2001). Autourde la modélisation en probabilités. Presses Universitaires de Franche-Comté.

Kaiser, G. (1996). Realitatsbezuge im Mathematikunterricht- Ein Uberblick uber die aktuelle und historische diskussion. EN Graumann, G.et al. (eds): Materialien fur einen realitatsbezogenen Mathematikunterricht, (pp. 66-84), Franzbecker. Bad Salzdetfurth.

Kaiser, G., Blum, W., Borromeo-Ferri, R., \& Stillman, G. (2011). Preface. In G. Kaiser, W. Blum, R. BorromeoFerri, \& G. Stillman (Eds.), Trends in 
teaching and learning of mathematical modelling: ICTMA14 (pp. 1-5). Springer.

Kuzniak, A. (2011). L'Espace de Travail Mathématique et ses genèses. Annales de Didactique et de Sciences Cognitives, 16, 9- 24.

Kuzniak, A., \& Richard, P. (2014). Spaces for mathematical work: Viewpoints and perspectives. Relime, 17(4.1), 17-26. DOI: https://dx.doi.org/10.12802/relime.13.1741a

Kuzniak, A., Nechache, A. \& Drouhard, J. P. (2016). Understanding the development of mathematical work in the context of the classroom. ZDM, 48(6), 861874. DOI: http://doi.org/10.1007/s11858-016-0773-0

Nechache, A. (2018). Le rôle des dimensions de l'ETM dans l'élaboration du travail mathématique dans de cadre de la résolution des tâches probabilistes. Menon. Journal of Educational Research, 4, 40-53.

Nechache, A. (2016). L'articulation des genèses de l'Espace de Travail Mathématique dans la ré- solution de tâches probabilistes faisant appel à la modélisation. En I. Gómez-Chacón, A. Kuzniak, K. Nikolantonakis, R. Richard y L. Vivier (Eds.), Acta del Quinto Simposio Internacional ETM (pp.157-166). University of Western Macedonia.

Montoya, E., \& Vivier, L.(2016). Mathematical Working Spaces as an analyzing tool for the teaching and learning of calculus. ZDM - International Journal on Mathematics Education, (5), 1689-1699. DOI: https://doi.org/10.1007/s11858-016-0777-9

Parzysz, B. (2011). Quelques questions didactiques de la statistique et des probabilités. Annalesde Didactique et de Sciences Cognitives,16, 127-147

Radford, G. (2008). The ethics of being and knowing: Towards a cultural theory of learning. In Radford, L., Schubring, G. \& Seeger, F. (Eds.). Semiotics in mathematics education epistemology, history, classroom, and culture (pp. 215-234). Sense Publishers. 
Verdugo,P.(2020). Un posible enfoque de complementariedad entre espacios de trabajo matem ático y modelización. UCMaule, 59, julio-diciem bre, 12-30. DOI: http://doi.org/10.29035/ucm aule.59.12

Rauscher, J. L. \& Adjiage, R.(2014). Espaces de travail de résolution d'un problème de modèlisation. Relime, 77(4), 47-64. DOI: http://dx.doi.org/10.12802/relime.13.1742

Verdugo-Hernández, P. (2020). Aproximación a la enseñanza de las sucesiones de números reales por medio de los Espacios de Trabajo Matemático. Revista Chilena de Educación Matemática, 12(2), 71-80. DOI: https://doi.org/10.46219/rechiem.v12i2.28 